\title{
Using Gene Genealogies to Detect Rare Variants Associated with Complex Traits
}

\author{
Kelly M. Burkett ${ }^{\mathrm{a}, \mathrm{c}, \mathrm{d}}$ Brad McNeney Jinko Graham $^{\mathrm{e}} \quad$ Celia M.T. Greenwood ${ }^{\mathrm{a}-\mathrm{c}}$ \\ ${ }^{a}$ Department of Epidemiology, Biostatistics and Occupational Health, and ${ }^{b}$ Division of Cancer Epidemiology, \\ Department of Oncology, McGill University, and ' Lady Davis Institute for Medical Research, Jewish General Hospital,

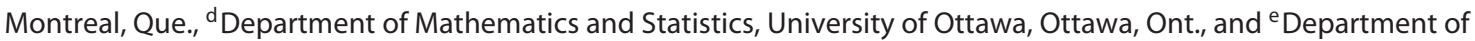 \\ Statistics and Actuarial Science, Simon Fraser University, Burnaby, B.C., Canada
}

\section{Key Words}

Gene genealogy - Rare variants - Association study .

Coalescent model $\cdot$ Region-based tests

\begin{abstract}
Background and Objective: Standard population genetic theory says that deleterious genetic variants are likely rare and fairly recently introduced. However, can this expectation lead to more powerful tests of association between diseases and rare genetic variation? The gene genealogy describes the relationships between haplotypes sampled from the general population. Although ancestral tree-based methods, inspired by the gene genealogy concept, have been developed for finding associations with common genetic variants, here we ask whether gene genealogies can help in identifying genomic regions containing multiple rare causal variants. Methods: With data simulated under several demographic models and using known gene genealogies, we developed and compared several tree-based statistics to determine which, if any, could detect the type of clustering expected with rare causal variants and whether the genealogic tree provides additional information about disease associations. Results and Conclusions: We found that a novel statistic based on the scaled distance between the tips of a tree performed better than other tree-based statistics. When
\end{abstract}

data were simulated with mild population growth, this statistic outperformed two standard non-tree-based methods, showing that an ancestral tree-based approach has potential for rare variant discovery.

(c) 2014 S. Karger AG, Basel

\section{Introduction}

Rare variants are increasingly being investigated for their role in the development of human traits and diseases. Sequencing studies have shown that the number of rare variants in the human genome is larger than previously expected [1-3]. Rare variants are also predicted to be more damaging than variants with larger minor allele frequencies (MAF) $[1,4]$. In addition, a number of recent studies have associated rare variants with common diseases. For example, Nejentsev et al. [5] found that 4 functional rare variants with a MAF $<3 \%$ in the IF1H1 gene were associated with type 1 diabetes, Guerreiro et al. [6] determined that a variant with a MAF of $0.3 \%$ in the TREM2 gene was associated with Alzheimer's disease, and Bonnefond et al. [7] detected that rare variants in the melatonin receptor gene $M T N R 1 B$ with a MAF $<0.1 \%$ contributed strongly to type 2 diabetes risk.

\section{KARGER}

E-Mail karger@karger.com

www.karger.com/hhe (c) 2014 S. Karger AG, Basel

0001-5652/14/0784-0117\$39.50/0 
As the single SNP-based association methods developed for common variants have low power with rare variants [8], analysis of sequencing data has spurred the development of new analysis methodologies for rare variant associations. By assuming that physically clustered rare variants - for example in the same gene - have effects on the same phenotype, most of these new methods involve some form of mathematical aggregation of rare variants physically clustered in a gene or defined region. For example, the CMC test of Li and Leal [8] collapses variants to indicate presence or absence of at least 1 rare variant at different MAF thresholds and tests for differences in the collapsed scores between cases and controls. As optimal MAF thresholds are unknown, the Variable Threshold (VT) test of Price et al. [9] maximizes a collapsed statistic over multiple MAF thresholds. These two methods assume that the effects of all variants in the collapsed score are in the same direction. Rare variant aggregation methods have also been developed that do not assume all risk or all protective variants [e.g. 10-14; for a recent review, see 15]. Drawbacks to rare variant aggregation approaches include setting MAF thresholds, deciding whether to include common SNPs, choosing which weighting scheme to use, and selecting the number of variants to be included in each statistic (the window size).

Individuals carrying the same disease-predisposing allele are likely to have inherited it from the same ancestor, and, therefore, at the disease-predisposing locus they are likely more closely related to each other than to individuals not carrying the allele. Instead of using the rare variant genotypes to group individuals, we can consider an alternative grouping based on the relatedness of individuals, which is available from the gene genealogy. The gene genealogy describes the relationships between sampled haplotypes. In the presence of recombination, the genealogy of a sample of haplotypes can be described by a set of trees giving the ancestral history of the sample at each locus in the region. Loci in intervals between historical recombination break points all have the same ancestral tree. Trees at nearby loci differ only in the portions of the tree affected by recombination, leading to correlations between trees at nearby loci.

We expect haplotypes from case individuals to tend to cluster together in the ancestral tree at a disease-predisposing locus. Many different tree-based approaches have been developed to detect regions associated with a disease due to a single common variant [e.g. 16-18]. In general, these approaches construct a tree or a set of trees consistent with the genotype data and use the predicted tree to define clusters. Cluster membership is then correlated with disease status. Such cluster-based approaches do not require knowledge of the true disease model (i.e., knowledge of the penetrance values is not required).

A likelihood approach is taken in the fine-mapping method of Larribe and Lessard [19] and the fine-mapping/ association method of Zöllner and Pritchard [20]. For these two methods, gene genealogies are sampled for sequences from case and control individuals based on population genetic models. The probability of a haplotype being from a case depends on whether the haplotype has the disease-predisposing variant - information that is provided by the genealogy and the disease model. The work of Adhikari et al. [21] extends the single-variant disease model of Zöllner and Pritchard [20] to multiple rare variants. The likelihood approaches of Larribe and Lessard [19], Zöllner and Pritchard [20], and Adhikari et al. [21] require specifying a disease model and superimposing disease mutations on the ancestries; the method of Adhikari et al. [21] also assumes that the disease-predisposing alleles at the rare variants are completely penetrant.

If there were multiple rare disease-predisposing variants in a region, the tree corresponding to each distinct causal rare variant locus would contain a small cluster of case haplotypes all carrying this mutation. However, as mentioned earlier, nearby trees in a small region are correlated due to the low chance of recombination events occurring in that region, and so the clustering of groups of tips in nearby trees is also correlated. Therefore, in the ancestral tree corresponding to a locus in a region containing multiple causal rare variants, we might expect to see multiple clusters of case haplotypes, with each cluster corresponding to a distinct causal mutation that occurred at a nearby locus. This is illustrated in figure 1, which shows three clusters corresponding to three different rare variant mutations in three different trees randomly sampled from a simulated region. To find a region with multiple causal variants, we therefore do not require the tree at exactly the location of each causal variant; instead, nearby trees capture the clustering of multiple causal variants. The amount of clustering of distinct rare variant mutations seen in a single tree will depend on the number and location of recombination events occurring in the history of the sample. Fortunately, since rare variants are more likely to be historically recent mutations, we expect few recombination events to have occurred within the rare variant clusters themselves, so that these clusters are likely to remain intact over small regions.

Based on the clustering in figure 1, we want to know whether tree-based statistics can detect an excess of small clusters of case haplotypes when there are multiple rare 
Fig. 1. Three ancestral trees for a sample of 50 haplotypes that were simulated using a coalescent model with a constant population size of 10,000 and a genomic region of a length of $20 \mathrm{~kb}$. These three trees were randomly sampled across the length of the region. The tip colors indicate haplotypes carrying 1 of 3 simulated rare variants. Despite the random selection of these trees' locations and the large differences in the tree shapes, all three trees show clustering of tips carrying the rare variants, as indicated by the clustering of each color in each tree.

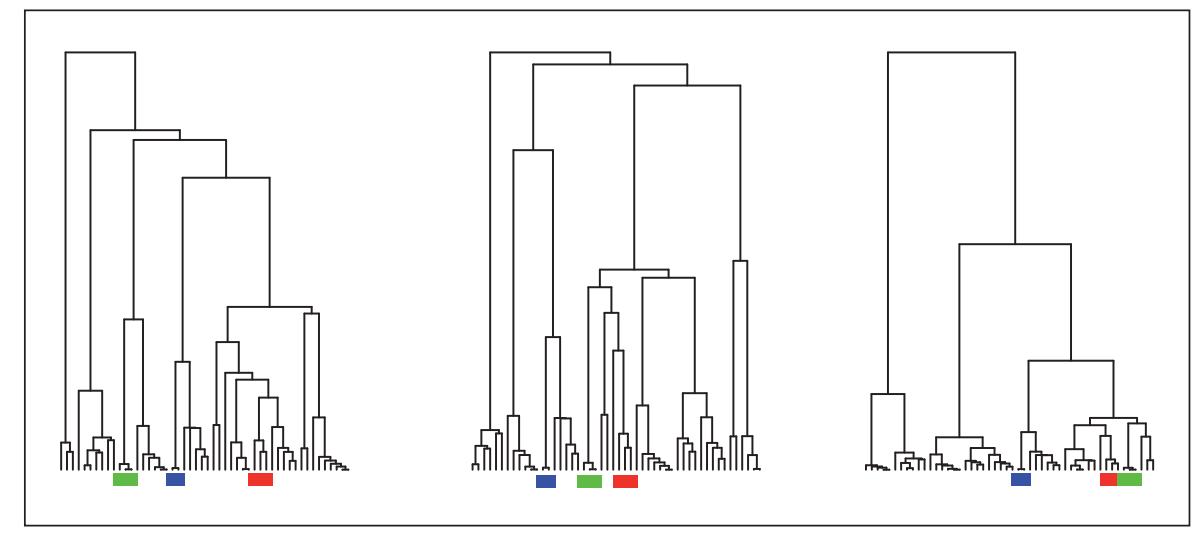

disease-associated variants in a region. Specifically, we are interested in test statistics that are disease model free, since the true disease model is not known in practice. We focus on a novel statistic that uses the tree to define distances between pairs of individuals and applies the Mantel test [22] to evaluate the correlation of the tree distance with the phenotype distance. Our use of the Mantel test is similar in spirit to haplotype-sharing statistics, which also correlate genetic and phenotypic similarity of haplotype pairs $[23,24]$. However, the tree is used to define genetic similarity rather than simply using the haplotypic states. There are potential advantages to using the ancestral tree to summarize relatedness of haplotypes rather than aggregating rare SNPs in a region. Specifically, we need not be concerned about how the results are affected by the decisions made on which MAF threshold to use, whether common variants should be included, or the number of SNPs in the collapsed statistic (the inclusion of non-causal SNPs adds 'noise' to the statistic).

In this article, we describe our investigation of the utility of a tree-based approach for rare variant identification. Although ancestral tree-based methods have been developed for finding associations with common genetic variants, here we ask whether gene genealogies can help in identifying regions containing multiple rare causal variants. We use a coalescent model to simulate haplotype and ancestral tree data under scenarios either with a constant population size or recent exponential growth.

We use multiple disease models to assign a disease status to haplotypes in the simulated population. Assuming known ancestral trees, we compare several tree-based statistics to determine which, if any, could detect the type of clustering expected with rare causal variants. We further compare our results to those of a single-locus and a region-based rare variant test to gain insight into whether

Using Gene Genealogies to Detect Rare Variants genealogical trees in the region with the causal loci provide information about disease associations over and above the information in the genotypes. We found that a novel statistic based on the scaled distance between the tips of the tree performed better than the other statistics evaluated, particularly when the underlying population has undergone recent exponential growth. Because we use the known trees, our results demonstrate a best-case scenario for rare variant mapping with tree-based approaches. Our rationale for using such a best-case scenario is that, if there are no gains to be made from considering a treebased approach when the tree is known, there is no point in pursuing further investigations incorporating tree uncertainty. Investigation into the effects of tree uncertainty and hence issues relating to how to estimate or predict the ancestral trees are not addressed in this work, but our results indicate that this is an area for future study.

\section{Methods}

We used simulated data to evaluate the ability of tree-based statistics to capture increased relatedness of multiple groups of cases. In this section, we first describe the coalescent simulations used to generate a sample of known ancestral trees and haplotypes and then describe how disease status was sampled based on the haplotype data. Next, we describe the tree-based statistics that we evaluated as well as the conventional association statistics that we compared to the tree-based approach. The comparison between the tree-based approach and the allele-based statistics is not entirely fair since the trees are not known in practice. Nevertheless, it is helpful for gaining insight about whether the trees provide additional information for finding rare causal mutations.

\section{Coalescent Simulations}

We used fastsimcoal [25] to simulate ancestral trees and haplotypes for a sample of 10,000 sequences in a small genomic region under scenarios with and without population growth. fastsimcoal 
Table 1. Settings for the exponential-growth demographic scenarios

\begin{tabular}{lllll}
\hline & $\begin{array}{l}\text { Present population } \\
\text { size, } N_{i} \text { (haplotypes) }\end{array}$ & $\begin{array}{l}\text { Growth rate, } r_{i} \\
(\% \text { per generation) }\end{array}$ & $\begin{array}{l}\text { Time, } t_{i} \\
\text { (generations) }\end{array}$ & Ref. \\
\hline Low & $1,000,000$ & 0.45 & 1,000 & Gravel et al. [28] \\
High & $4,000,000$ & 1.7 & 350 & Nelson et al. [3] \\
\hline
\end{tabular}

The model parameters are based on simplifications of the models estimated in the given references.

implements the sequentially Markov coalescent approximation [26] to the coalescent with recombination [27]. For the scenarios without population growth, the effective population size was set to 20,000 sequences. We used the 'SNP' data type setting of fastsimcoal to generate haplotypes of 2,000 SNPs in a region with a recombination rate of $1 \times 10^{-6}$ per generation per pair of adjacent SNPs. These settings were chosen to correspond to the per-generation mutation and recombination rates of approximately $3 \times 10^{-8}$ per base pair and $1 \times 10^{-8}$ per pair of adjacent base pairs, respectively. In a sample of 10,000 haplotypes from a haploid population of an effective size of 20,000 , the selected mutation rate leads to about one segregating site per 100 base pairs. Hence, the 2,000 SNPs are expected to span a region of approximately 200,000 base pairs.

To evaluate the effects of population growth, we used the 'DNA' setting of fastsimcoal to simulate datasets with variable numbers of SNPs from populations undergoing recent population growth. Specifically, we assumed that the population had been growing from an effective population size of approximately 10,000 sequences to the present effective size of $N_{i}$ for a period of $t_{i}$ generations at a growth rate of $r_{i}$ per generation. The selected values of $N_{i}, t_{i}$, and $r_{i}$ are given in table 1 and are simplifications of recent human population genetic models estimated from sequencing data $[2,3,28]$. Mutation and recombination rates were set to $3 \times$ $10^{-8}$ per base pair per generation and $1 \times 10^{-8}$ per pair of base pairs per generation, respectively, to match the models without growth. Due to the greater size of the datasets (many more SNPs and trees under growth), regions having a size of $100 \mathrm{~kb}$ were simulated.

\section{Sampling Case and Control Haplotypes}

Once the ancestral trees and haplotypes were simulated, the disease status was generated for each of the 10,000 haplotypes under a haploid disease model. In the rare variant models described below, the disease status was sampled based on the presence of causal SNPs on the haplotypes. SNPs in the middle $30 \mathrm{~kb}$ of the total region were eligible to be causal SNPs. In the null models, the disease status was sampled independently of the haplotype with a disease probability of 0.1 for all haplotypes.

For each dataset generated under one of the rare variant simulations, 10 causal SNPs were randomly sampled from the set of SNPs located in the gene region and having a MAF in the range of $0.5-1 \%$. We label this a type $i$ SNP selection strategy. If there were less than 10 SNPs in the MAF range in the gene region, the dataset was discarded and a new one was simulated. This occurred in approximately $1 \%$ of datasets. The disease probability was either $f_{0}$ if the haplotype had no causal alleles or $f_{\geq 1}$ if the haplotype had one or more causal alleles. The disease status was then sampled according to one of four different disease models, all having different ap- proximate disease prevalence, background penetrance values $\left(f_{0}\right)$ and phenocopy proportions (the proportion of case haplotypes having no causal alleles). The four sets of disease models are labelled M1 through M4 according to their approximate phenocopy proportion (table 2 ).

We also wanted to evaluate the effect of differing numbers of causal SNPs and MAF ranges on the rare variant approaches. We examined two additional strategies for selecting causal SNPs and labelled these type $i$ and type iii. Results with these additional SNP selection strategies are presented for disease model M3. The two additional SNP selection strategies are as follows: type $i i-4$ causal variants with a MAF in the range of $0.5-1 \%$ and 6 causal variants with a MAF in the range of $0.1-0.5 \%$, and type $i i i$ - no limit on the number of causal SNPs provided that they had a MAF $<1 \%$ and that the resulting disease prevalence was at least $K$. The combination of fixing desired values for prevalence, $f_{0}$, phenocopy proportions and SNP selection strategy (type $i-i i i)$ restricts the penetrance values of the causal alleles, $f_{\geq 1}$. Five different sets of penetrance values $\left(f_{0}, f_{\geq 1}\right)$ were required to achieve the desired prevalence/phenocopy proportions for the different methods of causal SNP selection.

Table 2 summarizes the main rare variant and null models that were run, the observed prevalence/phenocopy proportions, and the corresponding penetrance values for the scenarios without growth. Due to the longer run lengths of the growth scenarios, we could not run all disease models and therefore we focused on disease model M3.

After assigning disease status to the 10,000 sequences, 200 case and 200 control sequences were sampled from the affected and unaffected sequences. For each of the known ancestral trees simulated across the 100- or 200-kb regions, we then extracted the subtree corresponding to the sampled case and control sequences from the full ancestral tree of 10,000 tips. That is, we kept only those internal branches involving coalescence events between the 400 sampled sequences. For each combination of demographic and disease models, 200 datasets were simulated, each consisting of disease status, the haplotypes of the cases and controls, and the set of known subtrees.

Online supplementary table 1 (for all online suppl. material, see www.karger.com/doi/10.1159/000363443) provides summaries of the counts of case and control haplotypes carrying a causal allele across all causal variants in all datasets for the no- and low-growth scenarios. The median number of haplotypes carrying a causal variant is typically low due to the low MAF of the causal variants. The number also decreases as the penetrance of the causal allele decreases as well as for the models with no fixed limit on the number of causal variants. 
Table 2. Summary of disease models used to generate case/control data under the no-growth scenarios

\begin{tabular}{|c|c|c|c|c|}
\hline $\begin{array}{l}\text { Disease } \\
\text { model }\end{array}$ & $\begin{array}{l}\text { Prevalence } \\
K\end{array}$ & $\begin{array}{l}\text { Phenocopy } \\
\text { proportion }\end{array}$ & $\begin{array}{l}\text { SNP selection } \\
\text { strategy }\end{array}$ & Penetrance values \\
\hline Null & 0.10 & 1 & 0 causal loci & $f_{0}=f_{\geq 1}=0.1$ \\
\hline M1-i & 0.10 & 0.49 & 10 with a MAF of $0.5-1 \%$ & $f_{0}=0.05, f_{\geq 1}=0.8$ \\
\hline $\mathrm{M} 2-i$ & 0.10 & 0.70 & 10 with a MAF of $0.5-1 \%$ & $f_{0}=0.075, f_{\geq 1}=0.5$ \\
\hline M3-i & 0.10 & 0.75 & 10 with a MAF of $0.5-1 \%$ & $f_{0}=0.08, f_{\geq 1}=0.4$ \\
\hline M3-ii & 0.10 & 0.76 & 6 with a MAF of $0.1-0.5 \%$; 4 with a MAF of $0.5-1 \%$ & $f_{0}=0.08, f_{\geq 1}=0.6$ \\
\hline M3-iii & $0.10^{\dagger}$ & 0.74 & no limit on number; MAF $<1 \%$ & $f_{0}=0.08, f_{\geq 1}=0.4$ \\
\hline M4-i & 0.12 & 0.79 & 10 with a MAF of $0.5-1 \%$ & $f_{0}=0.1, f_{\geq 1}=0.4$ \\
\hline
\end{tabular}

Except where indicated, the prevalence and phenocopy proportion correspond to the mean values observed across 200 simulations.

$\dagger$ The prevalence for this disease model is fixed.

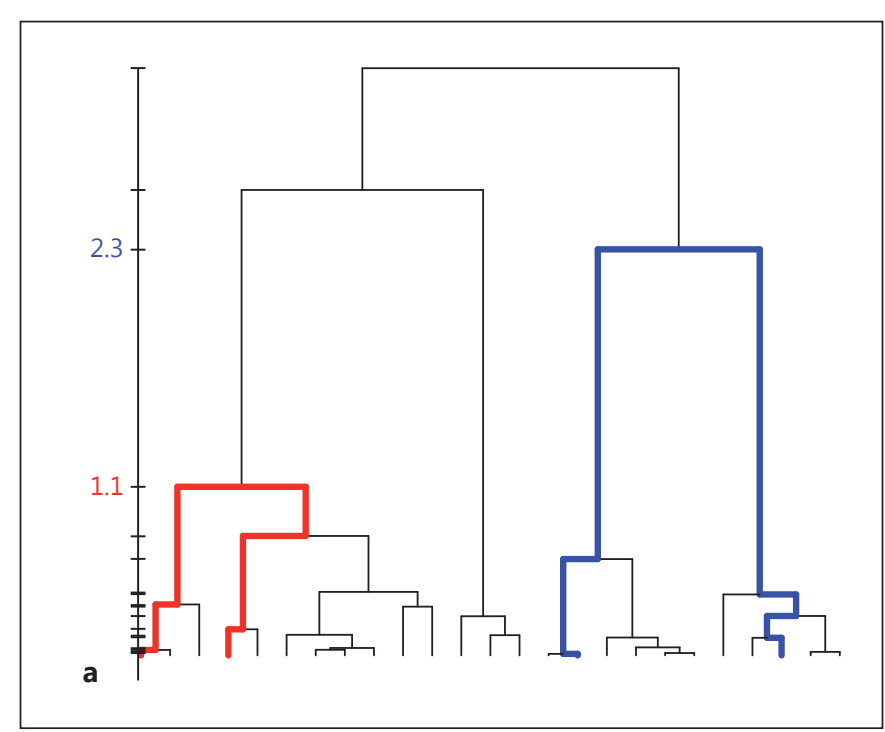

Fig. 2. Clusterings and distances illustrated on an example tree. a Using the branch length to define the distance between tips. The two haplotypes connected with the red path have their most recent common ancestor at ancestral distance 1.1, whereas the two blue

\section{Association Statistics}

The tree-based statistics that we examined can be separated into two classes: distance and cluster-based methods. As mentioned before, many cluster-based statistics have been investigated for detecting associations between disease status and a common variant. However, an association statistic based on the correlation of the tree distance to the phenotype distance has not yet been investigated.

We also compared the tree-based statistics to two commonly used allele-based statistics. In this section, we describe these statistics and explain how we use the disease and null model simulations to compare their distributions.

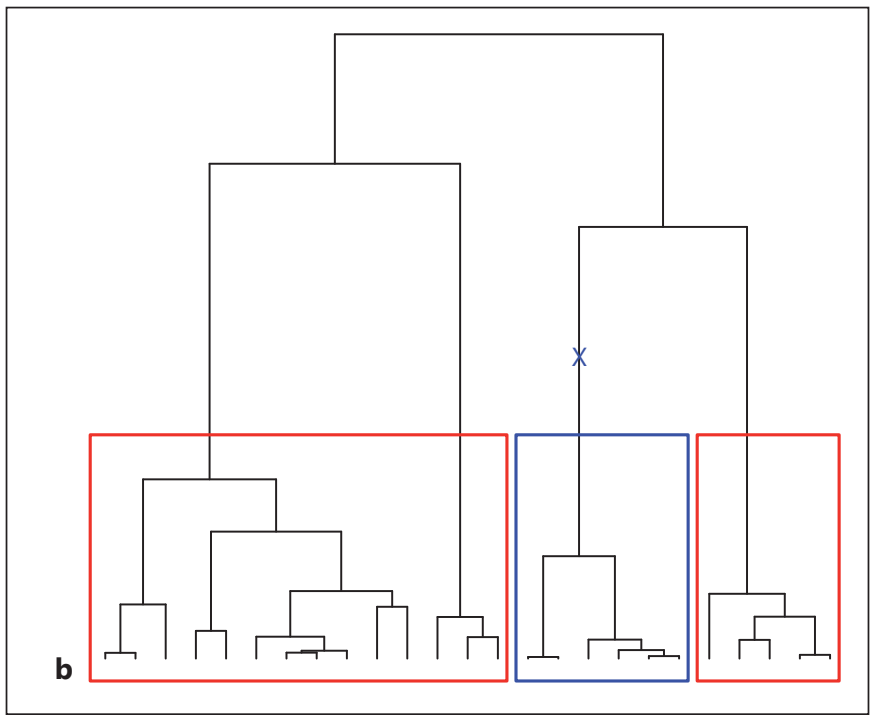

haplotypes have a coalescent distance of 2.3. b Minichiello-like clustering: an internal branch partitions the data into two sets, i.e. tips that descend from the branch marked with a blue ' $\mathrm{x}$ ' (blue box) and tips that do not (red boxes).

Distance-Based Tests

The tree can be used to define a distance matrix between pairs of tips, with distances based on the branch lengths separating the tips, as illustrated in figure $2 \mathrm{a}$. The tree distance-based approach is similar to haplotype-sharing statistics, except that the tree rather than the identity-by-state status of alleles is used to define the distance.

We evaluated three definitions of distance between two tips: (1) the time back to their first common ancestor; (2) the rank of the coalescence event for the first common ancestor proceeding back in time (i.e. all intercoalescence times are assigned to be 1 time unit), and (3) the mean-scaled time back to the first common an- 
cestor. For definition 3, the realized intercoalescence times are divided by their expected values based on the constant-size neutral coalescent. Under the constant-size neutral coalescent model, the intercoalescence time when there are $j$ branches is exponentially distributed with a rate $\left(\begin{array}{l}j \\ 2\end{array}\right)$. The rate of coalescence is therefore higher near the tips of the tree, leading to very short terminal branches. Conversely, the internal branch lengths near the root of the tree are much longer and have higher variability. By dividing the intercoalescence times by their expected values, we proportionally lengthen the contributions of the shorter terminal tip branches to the total tree time. Since we expect rare variants to have occurred very close to the terminal branches, the goal of this transformation is to increase the separation of the distances for the short branches near the tips of the tree.

Multiple statistics can then be used to evaluate whether cases are more closely related to each other than to non-cases. Since we expect that individuals who are close to each other on the tree will have similar phenotypes, we focused on the Mantel test to compute the correlation between the tree distance matrix and a phenotype distance matrix. We used a phenotype distance that is motivated by the similarity score of Beckmann et al. [23]: the similarity score between the $(i, j)^{\text {th }}$ pair is $s_{i j}=(y i-\mu)(y j-\mu)$, where $y_{i}$ is the phenotype ( 0 or 1 for case/control data) and $\mu$ is the prevalence. This score is bounded above by 1 because the phenotype measurements are 0 or 1 . As values close to 1 indicate similarity, the score was converted to a distance measure, $d_{i j}=1-s_{i j}$. We label the tests as MT, MT-r, and MT-mean for the Mantel tests based on the branch lengths, the ranks of the coalescence events, and the mean-scaled branch lengths, respectively.

\section{Cluster-Based Tests}

For cluster-based tests, the tree is used to define clusterings of the tip haplotypes; association between cluster membership and disease status is then tested with the Fisher exact test. Multiple different clusterings based on the tree are possible, but we focused on the approach used by Minichiello and Durbin [18], where clusters are based on the bipartitions created by each internal branch (fig. 2b). Each internal branch clusters the tip haplotypes into two groups: the group of tips that descend from that internal branch and the group of tips that do not. Some internal tips will lead to very small groups, and we therefore limit the eligible clusterings to bipartitions having a minimum of 20 tips, or $5 \%$ of the sample size. Since each internal branch induces a different clustering of the tips, the final statistic for a tree is the minimum $p$ value over the eligible clusterings of the Fisher exact test of association between cluster membership and disease status.

\section{Allele-Based Tests}

We also wanted to compare the tree-based approach to more conventional approaches in order to determine if under a best-case scenario the tree provides additional information about disease association over the genotypes at the rare causal loci. Single-locus association was assessed with the $p$ value of the Fisher exact test on a $2 \times 2$ contingency table of disease and allele status for each locus in each dataset.

We also included a popular rare variant test: the VT approach of Price et al. [9], implemented in the VTWOD function in the R package RVtests [29]. We chose this statistic because it does not require a fixed MAF threshold and is suited to the case of all rare variants having the same direction of effect, which corresponds with our simulated disease models. We applied the VT test across the simulated region using sliding windows of 50 SNPs overlapping by 25 SNPs.

Summarizing Results across Datasets and Test Statistics

The tree-based association statistics were computed on each of the known subtrees across the region. The single-locus results were computed for each SNP, and the VT test was evaluated for each of the windows across the region. For each of the test statistics evaluated, we used either the maximum across the region of the statistic or the maximum of $-\log _{10}$ of the p value as the score for the dataset.

For each scenario and each test statistic, we simulated $200 \mathrm{da}-$ tasets and computed the 200 maximum scores corresponding to these datasets. We are interested in comparing the distributions of the 200 scores across the different test statistics; however, the test statistics are not on the same scale, and the score for each dataset may be a maximum over a different number of tests. To make the score distributions comparable, we converted the scores to their corresponding empirical p values, defined as the proportion of observed values under the null model that are greater than or equal to the observed value under the alternative model. We obtained score values under the null model from our null scenario simulations. Therefore, the empirical null distributions used for computing empirical $p$ values account for the different number of tests performed on each dataset for each statistic. For example, for a rare variant scenario, the single-variant test is calculated at each polymorphic SNP, and therefore many more tests are performed on a dataset than are performed with the VT test. However, all maximized test statistics from the rare variant scenarios are compared to their maximum values from the null scenarios, thereby accounting for multiple testing. If formal testing procedures based on a given $\alpha$ level were performed, all tests would have the correct type 1 error rates.

A separate null scenario simulation was run for each of the population growth models. However, for a particular growth setting, a single null simulation with a disease prevalence set at $10 \%$ was used as the null distribution to compare to all disease models (M1-M4, types $i-i i i)$. For model M4, the prevalence of the disease was 0.12 rather than 0.1 , which means that the prevalence for the null distribution was slightly different. However, we would not expect this small difference in prevalence to have an effect on the results for model M4.

The distributions of empirical p values for the different test statistics were then compared. Under each scenario, we plotted the empirical cumulative distribution function (ECDF) of the empirical $\mathrm{p}$ values for the different test statistics. Since the ECDF at a point $x$ is the proportion of samples with an empirical $p$ value less than or equal to $x$, test statistics that tend to have lower empirical $\mathrm{p}$ values will have higher ECDF curves than the poorer-performing test statistics. Although they are not power curves, the ECDF curves do indicate the relative power of various test statistics.

\section{Computational Details}

Each simulated dataset was analyzed on a separate core of a cluster computer containing Intel Xeon $2.4 \mathrm{GHz}$ blades. Apart from simulating the genealogies using fastsimcoal (as described in the Coalescent Simulations section), all data processing and analyses were run using $\mathrm{R}$ (http://cran.r-project.org/). Manipulating and summarizing tree data was done using the $\mathrm{R}$ package ape [30]. To run all analyses for a single scenario, which included simulating 

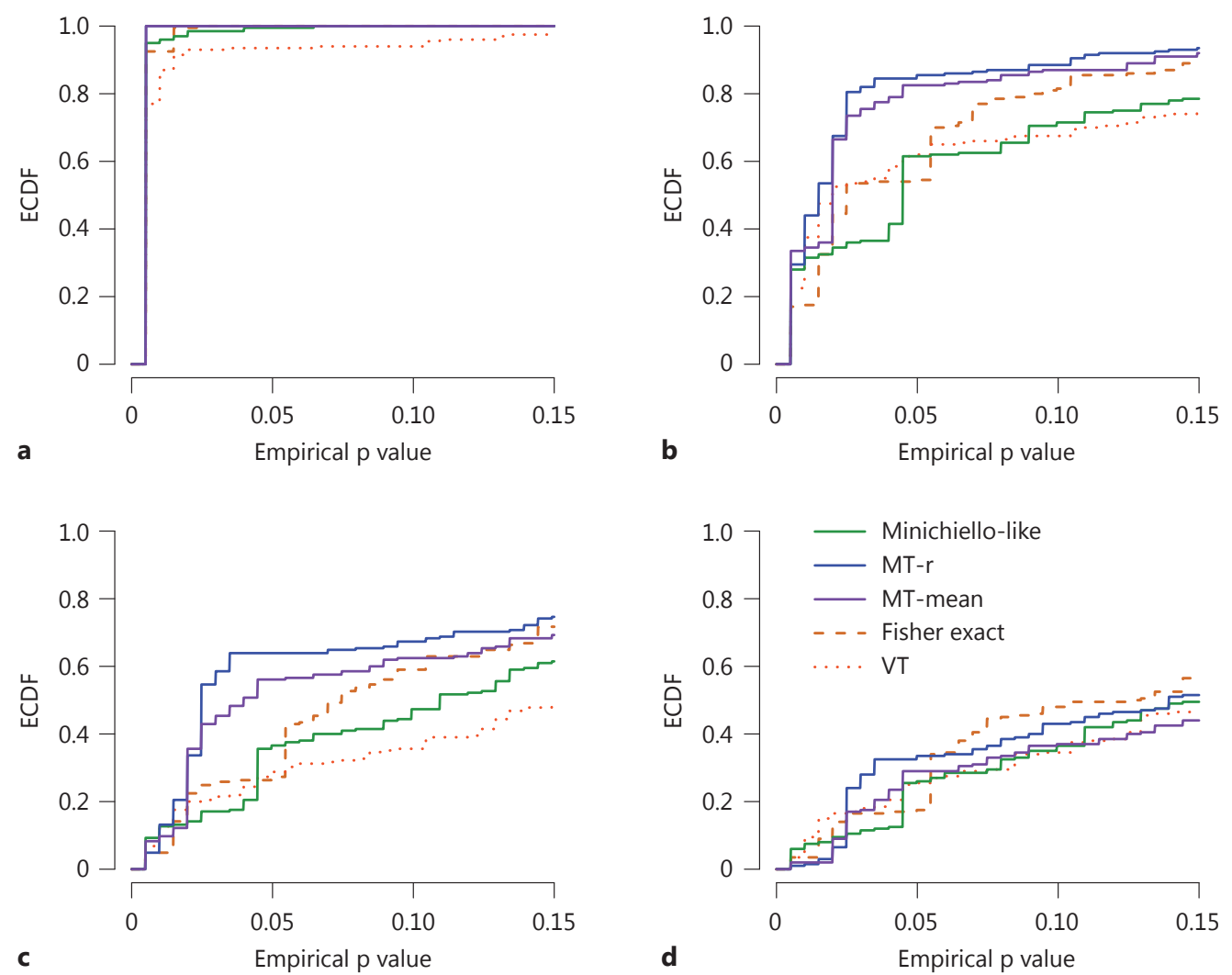

Fig. 3. ECDFs across 200 simulations of empirical $p$ values comparing five statistics: Fisher exact, VT, Minichiello-like, Mt-r and Mt-mean. Data were simulated with no population growth and assuming 10 rare variants (type $i$ ). For a better comparison between statistics, the $\mathrm{x}$-axis is shown only for $\mathrm{p}$ values $<0.15$. a Dis- ease model M1 $\left(f_{0}=0.05, f_{\geq 1}=0.8\right)$. b Disease model M2 $\left(f_{0}=0.075\right.$, $\left.f_{\geq 1}=0.5\right)$. c Disease model M3 $\left(f_{0}=0.08, f_{\geq 1}=0.4\right)$. d Disease model M4 $\left(f_{0}=0.1, f_{\geq 1}=0.4\right)$. The phenocopy proportions for disease models M1-M4 are $0.49,0.70,0.75$ and 0.79 , respectively. the genealogies, sampling the haplotypes and disease status, computing all association statistics, computing empirical $p$ values, and summarizing results, required approximately 1 week of computational time.

\section{Results}

\section{Data Simulated without Population Growth}

Figure 3 shows the ECDFs of the empirical p values across the 200 simulations for a constant population size and disease models with 10 rare variants (type $i$ ). With disease model M1, where the phenocopy proportion is the lowest, the five presented statistics perform equally well (fig. 3a); that is, the empirical p values are all very low. This model provides no meaningful comparisons be- tween the different approaches, and we do not present additional M1 results. Conversely, when the phenocopy proportion is the highest (M4), all five statistics perform equally poorly (fig. $3 \mathrm{~d}$ ). For example, the proportion of $\mathrm{p}$ values $<0.05$ (an estimate of the power at level 0.05 ) is between 0.18 and 0.34 for the five approaches. We therefore also do not present additional results for disease model M4.

Differences between the statistics can be seen at intermediate phenocopy proportions (fig. 3b, c). The two transformed branch length Mantel statistics (MT-r and MT-mean) outperform the three other statistics; the distribution of $\mathrm{p}$ values tends to have smaller values with the Mantel tests, as indicated by the higher curves. MT-r and MT-mean performed comparably to each other, while the Mantel test with unscaled branch lengths (MT) did not 


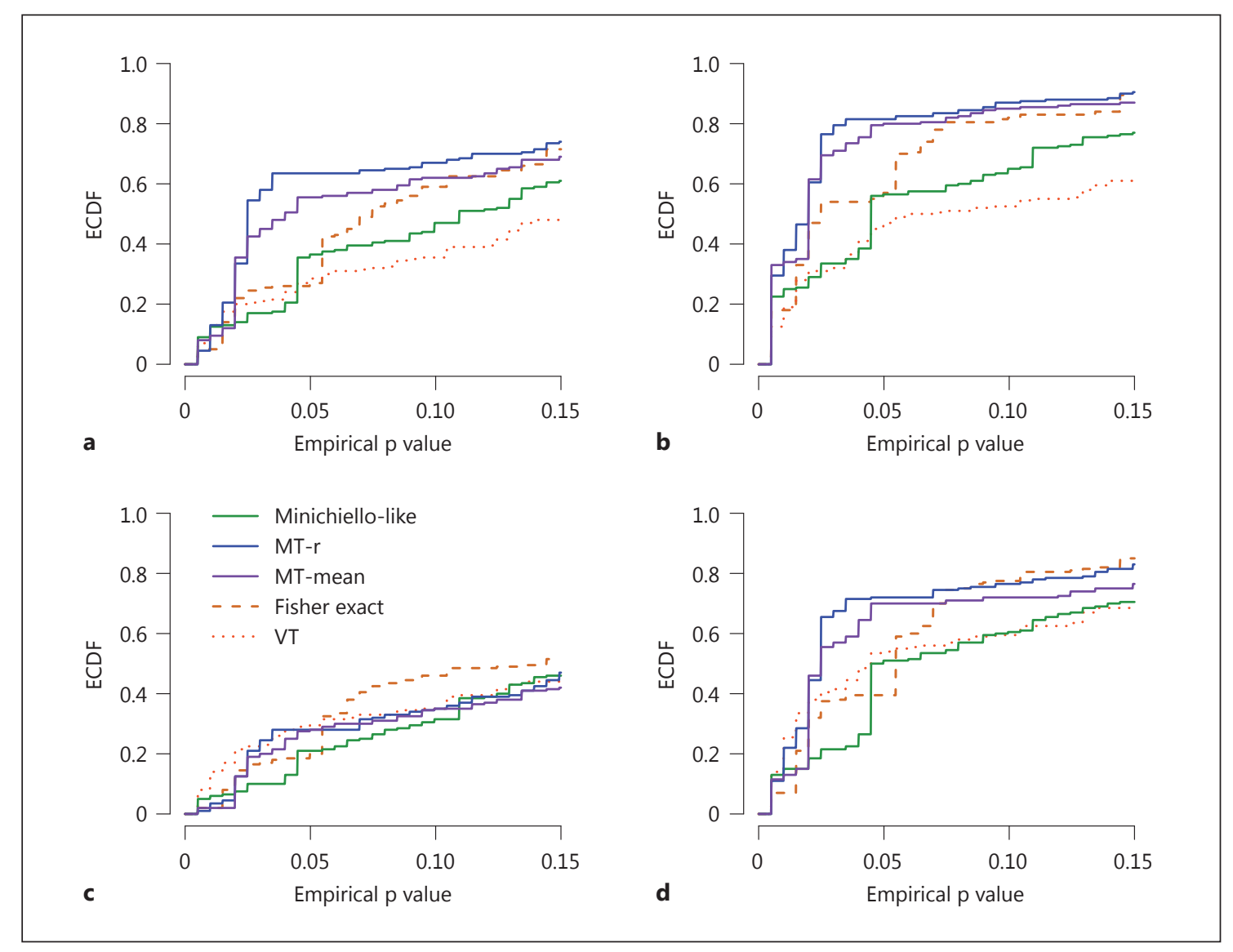

Fig. 4. ECDFs of empirical p values comparing results when different numbers of causal SNPs were chosen. Data were simulated with no population growth. a Ten causal loci with a MAF in the range of $0.5-1 \%(\mathrm{M} 3-i)$. b Four causal loci with a MAF in the range of $0.5-1 \%$ and six with a MAF in the range of $0.1-0.5 \%$ (M3-ii). c No limit on the number of causal loci (M3-iii). d No limit on the number of causal loci (M2-iii). perform well (not shown). The results with MT-r and MT-mean are also better than those with the two allelebased tests, indicating that there is additional information from the known trees about the presence of rare variants in the region.

Unexpectedly, the VT approach, an association test developed specifically for rare variants, did not perform well. In most cases, the single-locus tests performed as well as or better than the VT test.

Interestingly, the minimum p value of the single-locus tests occurs at one of the causal SNPs in only approximately $10 \%$ of datasets for disease models M1-i to M3-i and in $6 \%$ of datasets for M4-i. Generally, we would expect that the maximum association across SNPs should occur at the truly causal SNPs; however, it is possible that, with multiple disease-predisposing variants, a single SNP will capture the effect of multiple rare variants in a region [31].
In order to evaluate how the number and selection of causal SNPs influence the results, we examined two other SNP selection strategies (types $i$ and iii) and present the results with disease model M3 for these strategies in figure 4. For M3-ii (fig. 4b), when some of the causal SNPs have a lower MAF, we do not see changes in the ranking of the statistics; the two Mantel statistics continue to have lower $p$ values than the other statistics. However, when no limit is placed on the number of disease-predisposing SNPs, all methods perform very poorly under disease model M3 (fig. 4c). Performance using the model with no limit on the number of rare causal SNPs is similar to that of models with a fixed number of causal SNPs and higher phenocopy proportions (for example, see fig. 3d). We therefore also evaluated the no-limit scenario (type iii) with the lower phenocopy proportion disease model M2 (prevalence of $0.1, f_{0}=0.075, f_{\geq 1}=0.5$ ) and see a similar 


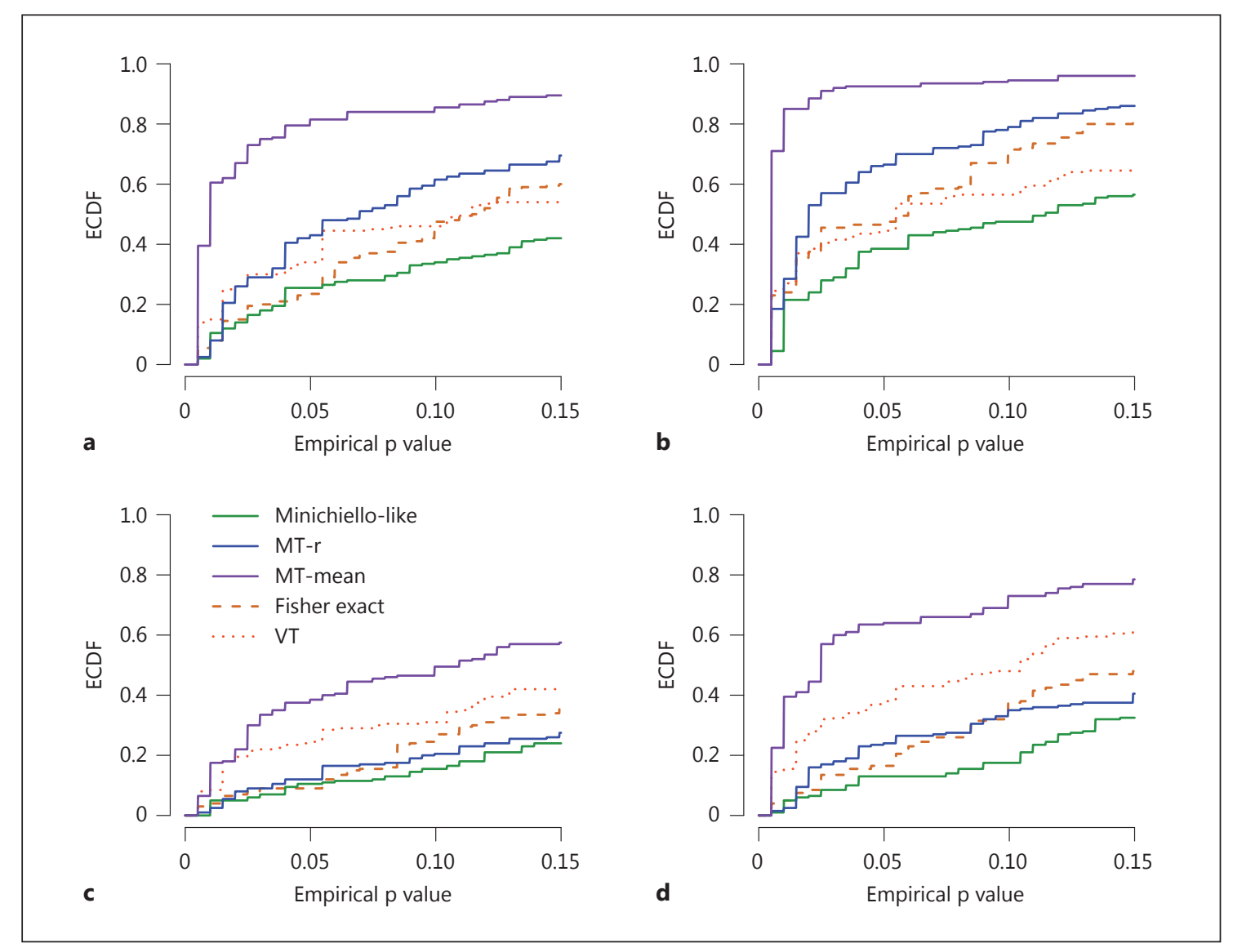

Fig. 5. ECDFs of empirical $p$ values for scenarios with a low rate of exponential growth. a Ten causal loci with a MAF in the range of $0.5-1 \%(\mathrm{M} 3-i)$. b Four causal loci with a MAF in the range of $0.5-$
$1 \%$ and six with a MAF in the range of $0.1-0.5 \%$. c No limit on the number of causal loci (M3-iii). d No limit on the number of causal loci (M2-iii). ranking of the statistics as with M3- $i$ and M3-ii (fig. $4 \mathrm{~d}$ vs. $4 a, b)$.

\section{Data Simulated with Population Growth}

Figure 5 shows the ECDFs of the empirical $p$ values for the scenarios simulated with the low rate of population growth and disease model M3. For all choices of the number of causal SNPs (types $i$-iii), the Mantel statistic with scaled branch lengths clearly performs better, with many smaller $p$ values, than the other statistics evaluated, including that of the rank-transformed Mantel test. In particular, the performance of the MT- $r$ is similar to that of the single-locus and Minichiello-like tests when there is no limit on the number of causal SNPs (fig. 5c, d).

Comparing the results of MT-mean with and without growth (fig. 4a, b vs. 5a, b), the distribution tends to have lower $p$ values when the data are simulated with growth and a fixed number of disease-predisposing variants (types $i$ and $i i$ ). For example, the proportion of $\mathrm{p}$ values $<0.05$ is 0.82 for mild growth versus 0.56 for no growth. Performance is best for the two scenarios where the number of causal SNPs is limited; however, even under no limit on the number of causal SNPs, MT-mean outperforms all other statistics. In addition, the difference between the ECDF curves for the MT-mean and VT tests is larger under scenarios with the low rate of growth.

There is a relative improvement in performance of the VT test over the single-locus test under the low rate of population growth when there is no limit on the number of rare causal variants. For example, when the number of causal SNPs is limited to 10 (type $i$ ), the single-locus test tends to produce $\mathrm{p}$ values that are roughly equal to or lower than those obtained with the VT test (fig. 5a, b). However, in the no-limit scenarios (type iii; fig. 5c, d), the VT test ranks second among the tests evaluated in terms of the proportion of low $\mathrm{p}$ values. These results can be 
explained by either an improved performance of the VT test or a worse performance of the single-locus test when there are many more causal SNPs. When the population has been growing, many more SNPs are rare or extremely rare than under models without growth. In the type iii simulations, the causal SNPs are randomly sampled from the SNPs with a frequency $<1 \%$. Therefore, under the growth model, the causal SNPs are more likely to be extremely rare, and each explains only a small proportion of the total prevalence. This leads to an increased number of causal SNPs in the $30-\mathrm{kb}$ region. The single-locus test on these extremely rare causal SNPs would be expected to have low power, whereas the VT test would be less affected because it aggregates genotypes for rare variants.

Online supplementary figure 1 shows ECDFs of the empirical $p$ values for the scenarios with the high rate of population growth. Model M3- $i$ having 10 rare variants with a MAF in the range of $0.5-1 \%$ was not run because many datasets had too few rare variants in the required MAF range; with the high rate of growth, most rare SNPs had a MAF $<0.5 \%$. The results are similar to those under the scenarios with a low growth rate. As with the nogrowth scenario, when there is no limit on the number of causal SNPs (type iii), the $\mathrm{p}$ values are all higher than when the number of causal SNPs is limited to 10 under disease model M3. When we look at the results under disease model M2-iii (online suppl. fig. S1c), MT-mean again outperforms the other statistics evaluated.

\section{Discussion}

We have presented an investigation of the utility of a tree-based association approach to the discovery of rare disease-predisposing variants. As a first step, we used the simplifying assumptions of known ancestral trees and a haploid disease model. We found that the novel Mantel statistic, which captures the correlation of the meanscaled tree distance to the phenotype distance, outperformed several other tree-based statistics for the disease models evaluated. Since the latest publications of population genetic data have estimated high rates of recent population growth [2], we included simulations with populations undergoing growth and found that a substantially better performance of the mean-scaled Mantel statistic was achieved in slowly exponentially growing populations.

The mean-scaled Mantel statistic computed on the known trees also had better results than the single-locus and VT tests, which are based on the genotypes at all loci in the region. With common causal variants, we would expect that the best information for association would come from the true causal locus and that if that locus was present in the dataset, the tree would not provide additional information about disease association. However, in the case of rare variants, we see that even with the genotypes at the true causal loci being included in the datasets, the true underlying gene genealogy contains additional information about the presence of the disease mutations.

Our investigation also provides a comparison of the rare variant method VT to the naive single-locus Fisher exact test. Interestingly, even though VT, a collapsingbased rare variant test, would be expected to be optimal for rare variants having unidirectional effects, for most simulation scenarios it did not perform much better than the single-locus test. Similar results are described in Kinnamon et al. [32], who found that methods using the minimum $\mathrm{p}$ value from the single-locus tests performed as well as or better than two popular collapsing-based statistics, even when corrected for multiple testing. We examined whether the poorer performance could be explained by our chosen window size and overlap by evaluating the performance of VT when both varied. Due to long run times, we only examined datasets simulated under the nogrowth demographic scenario with the M3-i disease model. As Brisbin et al. [33], we increased the window size from 10 to 80 SNPs, by 10 SNPs, with windows overlapping by $10 \%$ of SNPs, including the restriction that the overlap be at least 5 SNPs. We then used the minimum $p$ value across all window sizes as the score for the dataset. We also performed a VT analysis on the dataset corresponding to all SNPs in the $30-\mathrm{kb}$ gene region. Since the causal rare variants are only sampled from this $30-\mathrm{kb}$ region, this is the best window size for including all 10 causal variants. As shown in online supplementary figure 2, the VT results using the full gene are no better than the results obtained with a window size of 50 SNPs. However, the $\mathrm{p}$ values are lower using the window size-optimized VT procedure; the ECDF of the optimized approach is always higher than that of the non-optimized approach. However, the performance of the optimized approach is still not as good as that of the mean-scaled Mantel approach. For example, the proportion of $\mathrm{p}$ values $<0.05$ is 0.56 for the Mantel test and 0.43 for the optimized VT test (significant difference at the 0.05 level with the McNemar test). The minimum $\mathrm{p}$ value for the optimized VT approach was achieved with a window size of 20 for half of the datasets, indicating that window sizes smaller than 50 SNPs may be preferable for collapsing-based statistics. 
That the optimized VT approach tended towards smaller window sizes may be due to the sensitivity of the collapsing-based methods to the inclusion of non-causal loci in the collapsed statistic, as previously noted [34]. With larger window sizes, the number of causal variants remains fixed while the number of non-causal variants included in the collapsed statistic is increased.

In addition to a comparison of different approaches, our results are also informative about rare variant association in general. We found that when the disease model included a large number of rare variants, the distribution of empirical $p$ values tended to be larger for all statistics evaluated, and therefore all methods performed poorly. To compensate for the dilution of signal in disease models with very large numbers of causal variants, the phenocopy proportion must be lower.

To begin this consideration of the utility of a treebased approach, we used simplified models where the case status was assigned to the haplotypes. In reality, with diploid data, the case status would be assigned to the individual; we would not know which of an individual's two haplotypes actually carries disease-predisposing variants. If both haplotypes from an individual with a disease are assigned to the case status in the analysis, then it is possible that a haplotype without a disease-predisposing variant could be misclassified as a case if only one of the two haplotypes carried the causal variant. We therefore also ran simulations with 10 causal SNPs (type $i$ ) where the disease status was assigned based on the diploid genotype at the causal SNPs by randomly pairing the haplotypes. We compared the performance of the statistics in the haploid disease model simulations to their performance in diploid disease model simulations as follows. The rankings of the statistics were similar for the disease model with very high penetrance values (M1-i), the proportion of $\mathrm{p}$ values $<0.05$ was 0.81 for the mean-scaled Mantel statistic and 0.71 for Fisher exact and VT tests. However, overall performance was worse: under the same disease models, the distributions of empirical $p$ values tended to be higher with the diploid models than with the haploid models. Performance also dropped significantly as the penetrance of the rare variants decreased. For example, for M2- $i$ the proportion of simulations with $p$ values $<0.05$ ranged from 0.30 to 0.37 for all five test statistics.

In addition to simplified disease models, we also simplified the demographic models used to simulate the ancestral trees. Moreover, we did not simulate trees and haplotypes under scenarios that included selection. Rare variants are more likely to be functionally damaging [ 1 ,
4], and selection is more likely to act on polymorphisms with functional effects. However, the effect that selection would have on an ancestral tree-based approach is unclear. Classical population genetic results predict younger ages for deleterious alleles than for neutral alleles of the same frequency [35]. As deleterious alleles would have a low frequency due to purifying selection, haplotypes with causal and deleterious rare variants could be even more closely clustered on the tree due to the young age of the deleterious allele. Such increased clustering might be expected to increase the power of tree-based approaches. However, the prediction of younger ages of alleles undergoing selection has not been supported by more recent investigations involving alleles with a MAF $<1 \%$, possibly because the classical models do not adequately model a more complex human demographic history that includes bottlenecks and expansions [36].

The null simulations provide the distribution of the various statistics under the null hypothesis of no causal rare variants in the region. We measured the discrepancy between the distribution of the statistics under the null hypothesis to their distribution under different rare variant models by computing the distribution of empirical $p$ values calculated using the null simulations. As such, the tests would have the correct type 1 error rates if formal testing procedures based on an $\alpha=0.05$ cutoff were instead performed. Note, though, that we can simulate from the distribution under the null hypothesis only because we specified the population genetic and disease models used for simulating all the data, including the rare variant scenarios. A different empirical approach for sampling from the null distribution of the test statistic is the permutation of case/control labels; however, it would be better suited to an analysis of a single real dataset rather than multiple datasets simulated under a variety of known disease and demographic models, since permutation would need to be done on each dataset. Using the null simulations saves computational time as replicates from a single null simulation under a particular demographic model could be used as the null distribution for multiple disease models following the same demographic model with the same disease prevalence.

We focused our simulations on causal rare variants having a MAF in the range of $0.1-1 \%$ (models of type $i$ and $i$ ). We did not examine the impact of very rare variants (MAF $<0.1 \%$ ) on tree-based association statistics, although models of type iii would be expected to include some very rare variants. The Mantel-based statistic correlates the tree distance with the phenotype distance for pairs of tips. Therefore, to use this tree-based statistic, the 
MAF needs to be high enough so that the case haplotypes cluster into sets of pairs at a minimum. For example, if all causal rare variants were singletons, this tree-based statistic would not be able to detect association. For settings where the causal variants are expected to be very rare but the number of causal variants is expected to be high, aggregation-based approaches like the VT test may perform better.

Our simulations assumed that all causal rare variants had equal penetrance values regardless of their MAF. Lower-frequency variants are more likely to be non-synonymous [3] and potentially functional, and therefore it may be unrealistic to assume equal penetrances for all rare variants. However, the simulations with a fixed number of variants restricted attention to a limited range of MAFs, and therefore we would not expect more general schemes for assigning penetrance based on MAF to greatly affect the results. We would only anticipate larger sized clusters of cases for the less frequent rare variants. For the model with a fixed prevalence and no limit on the number of causal rare variants, we might see fewer causal rare variants in total since the very rare variants would have higher penetrances and would contribute more to the prevalence.

We did not know a priori which tree-based statistic would perform the best since it was unknown to what extent clustering of case haplotypes would persist in trees at locations other than the causal rare variant sites. We have therefore considered a number of different tree-based statistics and, for brevity, described only those approaches that performed reasonably well and provided interesting comparisons. Performance was poor for the clusterbased statistics; this was expected as they were developed under the assumption of a single or a small number of common variants. We also found that performance of the Mantel statistic was poor when using the actual branch lengths to measure tree distance. Transforming the branch times by their coalescence event rank or dividing by their expected mean was generally better. However, although the two transformations of the branch lengths led to statistics with comparable performance with data simulated assuming a constant-size coalescent model, the mean-scaled branch lengths greatly outperformed the rank-based transformation with data simulated assuming recent population growth. This interesting result highlights the need for investigation into optimal branch length transformations; it is possible that alternative branch length transformations could perform better under other, more complicated, disease models or demographic scenarios. We also only evaluated one measure of phenotype similarity, but alternative measures are possible. For example, we could create a similarity score that also gave concordantly unaffected haplotypes a higher value, which may perform better if some of the rare variants were protective. This work therefore establishes the potential of tree-based statistics, but further work would be required to optimize tree-based statistics to different disease and/or demographic models.

Finally, we assumed known trees when computing the tree-based statistics. Although this is obviously unrealistic, it provided a best-case scenario for evaluating whether tree-based statistics could pick up any clustering of cases due to multiple rare variants in a region. Others have also assumed known inheritance information to gain insight into the performance of proposed approaches under best-case, but unrealistic, scenarios. Browning and Thompson [37] assumed known inheritance information for their investigation into whether identity-by-descent (IBD) mapping could be applied to rare variants. They used a forward simulation strategy and defined pairs of samples as being IBD if they shared a common ancestor at 25 and 100 generations in the past. Smith and Kuhner [38] also assumed known trees when evaluating the optimal information available for fine-scale mapping using a likelihood-based approach. However, unlike our simulations, they were not evaluating rare variants - the minimum MAF was $>10 \%$.

For real data, the success of a tree-based method for identifying susceptibility variants will depend on two factors. The first is the ability to predict relatedness based on genotype data. The second is the relationship between relatedness and phenotype similarity. Here, we have presented an investigation of the second factor and have found that, even with rare susceptibility variants, the genealogical tree provides useful information about phenotypic similarity. Although the novel tree-distance approach performed well, computing the statistic requires knowledge of the ancestral tree and node times, which is not available in practice. A logical next step would therefore be to evaluate the effects of tree uncertainty (the first factor). Many of the tree-based approaches developed for common variants use the genotype data to impute a tree using phylogenetic or statistical algorithms. However, since these assume that the imputed tree is the true tree, tree uncertainty is not accounted for in the association test. An approach to incorporate this uncertainty is to sample genealogical trees conditional on observed genetic data using an MCMC-based genealogy sampler [e.g. 39]. However, MCMC-based approaches can be vulnerable to slow mixing for some datasets [e.g. 40]. Therefore, 
evaluating the performance of the tree-based statistics in the presence of tree uncertainty introduces the additional complications of evaluating the methods for estimating the trees, which is beyond the scope of this work. We expect that uncertainty in the tree will lead to decreased power of tree-based methods. However, if results are shown to differ greatly between analyses with known versus unknown ancestral trees, this would indicate that there are gains to be made by improving the tree sampling approaches through better algorithms or more data.

To summarize, we set out to determine if, with known trees, there were gains to be made from a tree-based approach for detecting rare variant associations. If no gains were observed, there would be no point in pursuing further investigations incorporating tree uncertainty. Our simulations have demonstrated that a test statistic which correlates the pairwise distances on the known ancestry with those in phenotypes outperforms two standard ge- notype-based approaches. We therefore conclude that tree-based approaches hold promise for detecting rare variants, and therefore future investigations that incorporate tree uncertainty are warranted.

\section{Acknowledgements}

This work was supported by the Natural Sciences and Engineering Research Council of Canada and the Canadian Institutes of Health Research operating grant MOP 115110. Some of the work was undertaken while J.G. and B.M. were visitors at the Lady Davis Institute; these authors would like to thank the Institute and in particular Dr. Celia Greenwood for their hospitality.

\section{Disclosure Statement}

The authors have no conflicts of interest to declare.

\section{References}

1 Coventry A, Bull-Otterson LM, Liu X, Clark AG, Maxwell TJ, Crosby J, Hixson JE, Rea TJ, Muzny DM, Lewis LR, Wheeler DA, Sabo A, Lusk C, Weiss KG, Akbar H, Cree A, Hawes AC, Newsham I, Varghese RT, Villasana D, Gross S, Joshi V, Santibanez J, Morgan M, Chang K, Iv WH, Templeton AR, Boerwinkle E, Gibbs R, Sing CF: Deep resequencing reveals excess rare recent variants consistent with explosive population growth. Nat Commun 2010;1:131.

-2 Keinan A, Clark AG: Recent explosive human population growth has resulted in an excess of rare genetic variants. Science 2012;336:740743.

-3 Nelson MR, Wegmann D, Ehm MG, Kessner D, St Jean P, Verzilli C, Shen J, Tang Z, Bacanu SA, Fraser D, Warren L, Aponte J, Zawistowski M, Liu X, Zhang H, Zhang Y, Li J, Li Y, Li L, Woollard P, Topp S, Hall MD, Nangle K, Wang J, Abecasis G, Cardon LR, Zöllner S, Whittaker JC, Chissoe SL, Novembre J, Mooser V: An abundance of rare functional variants in 202 drug target genes sequenced in 14,002 people. Science 2012;337: 100-104.

4 Gorlov I, Gorlova O, Frazier M, Spitz M, Amos C: Evolutionary evidence of the effect of rare variants on disease etiology. Clin $\mathrm{Ge}-$ net 2011;79:199-206.

5 Nejentsev S, Walker N, Riches D, Egholm M, Todd JA: Rare variants of IFIH1, a gene implicated in antiviral responses, protect against type 1 diabetes. Science 2009;324:387-389.
6 Guerreiro R, Wojtas A, Bras J, Carrasquillo M, Rogaeva E, Majounie E, Cruchaga C, Sassi C, Kauwe JS, Younkin S, Hazrati L, Collinge J, Pocock J, Lashley T, Williams J, Lambert JC, Amouyel P, Goate A, Rademakers R, Morgan K, Powell J, St George-Hyslop P, Singleton A, Hardy J; Alzheimer Genetic Analysis Group: TREM2 variants in Alzheimer's disease. N Engl J Med 2013;368:117-127.

7 Bonnefond A, Clément N, Fawcett K, Yengo L, Vaillant E, Guillaume JL, Dechaume A, Payne F, Roussel R, Czernichow S, Hercberg S, Hadjadj S, Balkau B, Marre M, Lantieri O, Langenberg C, Bouatia-Naji N, Charpentier G, Vaxillaire M, Rocheleau G, Wareham NJ, Sladek R, McCarthy MI, Dina C, Barroso I, Jockers R, Froguel P: Rare MTNR1B variants impairing melatonin receptor $1 \mathrm{~B}$ function contribute to type 2 diabetes. Nat Genet 2012; 44:297-301.

-8 Li B, Leal SM: Methods for detecting associations with rare variants for common diseases: application to analysis of sequence data. Am J Hum Genet 2008;83:311-321.

-9 Price AL, Kryukov GV, de Bakker PIW, Purcell SM, Staples J, Wei LJ, Sunyaev SR: Pooled association tests for rare variants in exon-resequencing studies. Am J Hum Genet 2010; 86:832-838.

10 Han F, Pan W: A data-adaptive sum test for disease association with multiple common or rare variants. Hum Hered 2010;70:42-54.

11 Neale BM, Rivas MA, Voight BF, Altshuler D, Devlin B, Orho-Melander M, Kathiresan S, Purcell SM, Roeder K, Daly MJ: Testing for an unusual distribution of rare variants. PLoS Genet 2011;7:e1001322.
12 Wu M, Lee S, Cai T, Li Y, Boehnke M, Lin X: Rare-variant association testing for sequencing data with the sequence kernel association test. Am J Hum Genet 2011;89:82-93.

13 Lee S, Emond MJ, Bamshad MJ, Barnes KC, Rieder MJ, Nickerson DA, Christiani DC, Wurfel MM, Lin X: Optimal unified approach for rare-variant association testing with application to small-sample case-control wholeexome sequencing studies. Am J Hum Genet 2012;91:224-237.

14 Sun J, Zheng Y, Hsu L: A unified mixed-effects model for rare-variant association in sequencing studies. Genet Epidemiol 2013;37: 334-344.

15 Burkett KM, Greenwood CM: A sequence of methodological changes due to sequencing. Curr Opin Allergy Clin Immunol 2013;13: 470-477.

16 Durrant C, Zondervan KT, Cardon LR, Hunt S, Deloukas P, Morris AP: Linkage disequilibrium mapping via cladistic analysis of singlenucleotide polymorphism haplotypes. Am J Hum Genet 2004;75:35-43.

17 Bardel C, Danjean V, Hugot J, Darlu P, Génin E: On the use of haplotype phylogeny to detect disease susceptibility loci. BMC Genet 2005;6:24.

18 Minichiello MJ, Durbin R: Mapping trait loci by use of inferred ancestral recombination graphs. Am J Hum Genet 2006;79:910-922.

19 Larribe F, Lessard S: A composite-conditional-likelihood approach for gene mapping based on linkage disequilibrium in windows of marker loci. Stat Appl Genet Mol Biol 2008; 7:Article27. 
20 Zöllner S, Pritchard JK: Coalescent-based association mapping and fine mapping of complex trait loci. Genetics 2005;169:1071-1092.

-21 Adhikari K, AlChawa T, Ludwig K, Mangold E, Laird N, Lange C: Is it rare or common? Genet Epidemiol 2012;36:419-429.

22 Mantel N, Valand RS: A technique of nonparametric multivariate analysis. Biometrics 1970;26:547-558.

23 Beckmann L, Thomas DC, Fischer C, ChangClaude J: Haplotype sharing analysis using mantel statistics. Hum Hered 2005;59:67-78.

24 Allen AS, Satten GA: Association mapping via a class of haplotype-sharing statistics. BMC Proc 2007;1(suppl 1):S123.

-25 Excoffer L, Foll M: Fastsimcoal: a continuoustime coalescent simulator of genomic diversity under arbitrarily complex evolutionary scenarios. Bioinformatics 2011;27:13321334.

26 McVean GA, Cardin NJ: Approximating the coalescent with recombination. Philos Trans R Soc Lond B Biol Sci 2005;360:1387-1393.

27 Hudson RR: Gene genealogies and the coalescent process; in Futuyama D, Antonovics J (eds.): Oxford Surveys in Evolutionary Biology. Oxford, Oxford University Press, 1990, vol 7.
28 Gravel S, Henn BM, Gutenkunst RN, Indap AR, Marth GT, Clark AG, Yu F, Gibbs RA, Bustamante CD: Demographic history and rare allele sharing among human populations. Proc Natl Acad Sci USA 2011;108: 11983-11988.

$29 \mathrm{Xu}$ C, Ladouceur M, Dastani Z, Richards JB, Ciampi A, Greenwood CMT: Multiple regression methods show great potential for rare variant association tests. PLoS One 2012; 7:e41694.

30 Paradis E, Claude J, Strimmer K: APE: analyses of phylogenetics and evolution in R language. Bioinformatics 2004;20:289-290.

31 Dickson SP, Wang K, Krantz I, Hakonarson $\mathrm{H}$, Goldstein DB: Rare variants create synthetic genome-wide associations. PLoS Biol 2010;8:e1000294

32 Kinnamon DD, Hershberger RE, Martin ER: Reconsidering association testing methods using single-variant test statistics as alternatives to pooling tests for sequence data with rare variants. PLoS One 2012;7:e30238.

33 Brisbin A, Jenkins GD, Ellsworth KA, Wang L, Fridley BL: Localization of association signal from risk and protective variants in sequencing studies. Front Genet 2012;3:173.

34 Pongpanich M, Neely ML, Tzeng JY: On the aggregation of multimarker information for marker-set and sequencing data analysis: genotype collapsing vs. similarity collapsing. Front Genet 2012;2:110.
5 Maruyama T: The age of a rare mutant gene in a large population. Am J Hum Genet 1974; 26:669-673.

- 36 Maher MC, Uricchio LH, Torgerson DG, Hernandez RD: Population genetics of rare variants and complex diseases. Hum Hered 2012;74:118-128.

37 Browning SR, Thompson EA: Detecting rare variant associations by identity by descent mapping in case-control studies. Genetics 2012;190:1521-1531.

38 Smith LP, Kuhner MK: The limits of finescale mapping. Genet Epidemiol 2009;33: 344-356.

39 Burkett KM, McNeney B, Graham J: A Markov chain Monte Carlo sampler for gene genealogies conditional on haplotype data; in Chaubney Y (ed.): Some Recent Advances in Mathematics and Statistics, Proceedings of Statistics 2011 Canada/IMST 2011-FIM XX, Montreal, July 2011. Singapore, World Scientific Publishing, 2013, pp 29-44.

40 Burkett KM, McNeney B, Graham J: Markov chain Monte Carlo sampling of gene genealogies conditional on unphased SNP genotype data. Stat Appl Genet Mol Biol 2013;12:559581 